\title{
Numerical modeling of slippage and adsorption effects on gas transport in shale formations using the lattice Boltzmann method
}

Yang Ning ${ }^{\mathrm{a}}$, Yang Jiang ${ }^{\mathrm{a}, \mathrm{b}}$, and Guan Qin ${ }^{\mathrm{a}, \dagger}$

a: Petroleum Engineering Program, Department of Chemical \& Biomolecular Engineering, University of Houston, Houston, TX 77204, USA

b: SinoFTS Petroleum Services Ltd., Beijing 100101, China

†Corresponding author: gqin@uh.edu, +1 (713) 743-5415

\begin{abstract}
Shale formations consist of numerous nanoscale pores within a range of $2 \mathrm{~nm}-50 \mathrm{~nm}$; the shale gas flow within this size range under typical shale reservoir pressure and temperature will fall into the slip flow or the transitional flow regime $0.001<K_{n}<10$. Besides nano-pores in shale, there exist a number of mesoscopic and macroscopic pores with size larger than $50 \mathrm{~nm}$, and many micrometer fractures. Natural gas, mainly methane, flows through nanoscale pores, mesoscale, and macroscopic pores or fractures during the production period. Gas slippage described by the Klinkenberg effect reduces viscous drag near the pore walls and influences permeability. In shale, the majority of gas molecules are adsorbed in kerogen that is considered to be organic source rocks. Within nano-pores and meso-pores, Darcy's law cannot effectively describe this type of transport phenomena due to its continuum assumption. Alternatively, the kinetic-based lattice Boltzmann method (LBM) becomes a strong candidate for simulating an organic-rich shale reservoir that contains a large number of nano-pores. In this paper, we present a multiple-relaxation-time (generalized) LBM, which is considered to be one of the most efficient LBM models. For gas flow in a confined system, its molecular mean free path is corrected depending on the size of the confined system and the distance of the gas molecules from the pore walls. Gas slippage on pore walls is captured with a combined bounce-back specular reflection boundary condition. In addition, adsorbed gas in shale has a significant influence on gas transport in shale gas production. Here, we propose to incorporate inter-molecular and adsorptive forces into the generalized LBM algorithm to capture gas adsorptions in organic nano-pores. Therefore, this approach is able to simulate gas flow with adsorption effect. Many factors are believed to control the flow mechanisms in these types of pores, including the pore size distribution, the specific surface area, and the adsorptive feature of the pore walls. The simulation results agree well with the existing data for high Knudsen flows between 2D parallel plates. Accounting for the adsorption and slippage effects, flow phenomena are investigated by varying different controlling factors in both simple and complex structures. The permeability of methane is also determined for complex porous geometries.
\end{abstract}


Keywords: Shale gas, slippage, adsorption, lattice Boltzmann, permeability.

\section{Introduction}

Shale gas is becoming a significant source of unconventional natural gas. The production of shale gas mainly depends on its characteristics, including its pore size distribution, organic richness, natural/factitious fractures, etc. A significant portion of shale gas is stored in nano-pores within kerogen pockets that are $200 \mathrm{~nm}$ to $500 \mathrm{~nm}$ in size, and nano-pores have sizes in the range of $2 \mathrm{~nm}-50 \mathrm{~nm}$ (Ambrose et al., 2010; Adesida et al., 2011; Wei et al., 2013; Kang et al., 2011). Consequently, it is essential to understand the natural gas flow mechanisms in nano-pores in order to predict long-term shale gas production and shale gas reserves. In nano-pores, a dimensionless number, the Knudsen number $\left(K_{n}\right)$, is important for describing the flow mechanisms. The Knudsen number is defined as the ratio of the molecular mean free path $\lambda$ to a representative physical length of the interest $L$, which is the representative diameter of nano-pores. The molecular mean free path is the average distance that a molecule travels between two successive collisions. It is a function of pressure, temperature, and the type of gas molecules. With different Knudsen numbers, the flow regime falls into the following four categories: continuum flow $\left(K_{n}<0.001\right)$, slip flow $\left(0.001<K_{n}<0.1\right)$, transitional flow $\left(0.1<K_{n}<\right.$ $10)$, and free molecular flow $\left(K_{n}>10\right)$. Natural gas flow in nano-pores is considered to be either a slip flow or a transitional flow regime, depending on pore condition, size of nano-pore and type of gas molecules. In both of these flow regimes, the continuum assumption of fluid flow breaks down and Navier-Stokes (N-S) equations, with a regular no-slip boundary condition, cannot be applied because the gas slippage effect becomes significant. A large portion of natural gas in shale formations is claimed to be adsorbed in kerogen pockets because of the organic nature of kerogen (Kang et al., 2011), so we must take the adsorption/desorption effect into account to effectively describe the mechanism and storage of gas flow in shale formations. Permeability is a key parameter for describing the flow characteristics of hydrocarbons in oil and gas reservoirs. In shale formations, Darcy's law cannot be used to characterize permeability as it has been derived from N-S equations via homogenization (Whitaker, 1986). Accurately determining permeability can significantly improve the prediction of production in either numerical reservoir simulations or empirical methods. In gas reservoirs, the slippage of gas molecules at the pore wall gives apparently higher permeability than would be obtained by liquid measurements, which is known as the Klinkenberg effect (Klinkenberg, 1941). This effect may have a significant impact on gas flow behavior, especially in low permeability media. Klinkenberg stated that effective gas permeability is a pressure-dependent property and it is a linear function of the reciprocal mean pressure. Shale reservoirs have different characteristics from conventional gas reservoirs, and the permeability of shale is dependent of multiple conditions, such as pore pressure, temperature and organic content, and so on. Therefore, 
different modified Klinkenberg slippage theories have been proposed based on higher-order dependency between gas permeability and pore pressure (Moghadam and Chalaturnyk, 2014; Fathi et al., 2012; Ziarami and Aguilera, 2012).

The numerical modeling of gas flow in organic nano-pores requires proper numerical techniques. The Boltzmann equation is well-accepted for modeling gas flows across all types of flow regimes. The lattice Boltzmann equation is a discretized form of the continuous Boltzmann equation and it models the distribution function of fluid particles. Two types of lattice Boltzmann method (LBM) models include the single-relaxation-time model, based on the Bhatnagar-Gross-Krook (BGK) approximation (Bhatnagar et al., 1954), and the multiple-relaxation-time (generalized) model (d'Humieres, 1992). Generalized LBM relaxes various moments (i.e., density, energy, momentum, heat flux, stress tensor, etc.) to their equilibrium states at different relaxation times during collisions, which provides great advantages regarding its theories, parameter selections, and numerical stability over the BGK LBM (Lallemand and Luo, 2000). Standard LBM is a second order approximation of the continuous Boltzmann equation and it has the same accuracy with N-S equations as the continuous Boltzmann equation. LBM and N-S equations have been successfully applied to modern computational fluid dynamics for thermodynamic quasi-equilibrium flows. However, their standard formulations are inadequate to simulate extremely low speed flows with high Knudsen numbers (i.e., $K_{n} \gg 0.001$ ) in which the fluids are in a thermodynamic non-equilibrium state (Meng et al., 2012). For high Knudsen flows, the failure of the standard LBM can be attributed to its insufficient ability to describe the Knudsen layer (a kinetic boundary layer over a solid surface), and its thickness is comparable to gas molecular mean free path (Guo et al., 2006; Shan et al., 2006). Within the Knudsen layer, the thermodynamic quasi-equilibrium state cannot be achieved due to insufficient collisions between the gas molecules, and gas slippage cannot be ignored. Therefore, a more effective LBM model to capture gas slippage within the Knudsen layers is needed.

In the past decade, LBM has been widely used in modeling high Knudsen flow due to its theoretical advantages over N-S equations. In a high Knudsen flow, the kinetic Knudsen layer is a dominant portion of the whole flow region and the slippage effect becomes important. The failure to characterize the Knudsen layer can significantly influence the ability to model the flow mechanisms. Modifying the gas mean free path in the Knudsen flow region has been proven to be able to improve the flow prediction (Guo et al., 2006; Shan et al., 2006; Zhang et al., 2006; Niu et al., 2007; Sbragaglia and Succi, 2005; Guo et al., 2007; Guo et al., 2008). One of the relaxation times in generalized LBM is related to the gas molecule's mean free path or the fluid viscosity, so this relaxation time needs to be adjusted to capture flow behavior within the Knudsen layers. Moreover, a proper boundary condition that can accurately describe the gas slippage on solid boundaries is required. The most widely used non-slip bounce-back 
boundary condition in LBM, which strictly guarantees the conservation of mass and momentum, cannot be applied for high Knudsen flows. Therefore, Zhang et al. (2006) proposed an effective mean free path with a diffuse scattering boundary condition for high Knudsen flows. Niu et al. (2007) and Suga et al. (2010) applied higher order moments and an effective relaxation time to model the Knudsen layer. Guo et al. $(2007 ; 2008)$ proposed an extended Navier-Stokes formulation based on an effective mean free path and applied it into the generalized LBM with a combination of bounce-back and specular reflection (CBBSR) boundary conditions (Sbragaglia and Succi, 2005). The combination of bounce-back and specular reflection is used to describe the slippage on the solid boundary, while the portion of the specular reflection is found related to the effective relaxation time (Guo et al., 2008). Chen and Tian (2009) proposed another boundary condition based on the Langmuir slip model.

Another important issue in shale gas transport is the adsorption effect, as a significant amount of natural gas stored in shale formations is absorbed gas that can be quantified using Langmuir isotherms (Kang et al., 2011). Shale gas transport can be dominated by the release of absorbed gas from the nanopores. The hopping of gas molecules between the adsorbed gas and the free gas requires an intermolecular force. Shan and Chen (1993) have introduced an intermolecular potential among different phases, which adds an attraction or repulsion to the elastic collisions. Sukop and Or (2004) have introduced an intermolecular potential between solid walls and fluid particles to capture the adsorption effect based on Shan and Chen's work (Shan and Chen, 1993). Fathi et al. (2012) first applied the adsorptive force onto LBM to capture adsorbed gas in kerogen capillary tubes. Since intermolecular potentials are incorporated into LBM, the equation of state (EOS) needs to be modified accordingly (He and Doolen, 2002; Sukop and Thorne Jr., 2006) in order to include the non-ideality effect. By accounting for the adsorptive/cohesive forces among particles, we can capture the adsorption and interphase phenomenon. Recently, Ren et al. (2015) calculated the transports of the adsorbed gas and the free gas separately. The transport of the adsorbed gas was solved by the surface diffusion theory, where the amount of the adsorbed gas was quantified by the Langmuir isotherm. In the meantime, transport of the free gas was solved by the non-ideal LBM method.

In this paper, we first discuss the generalized LBM with an effective relaxation time associated with the effective mean free path of the gas. This effective mean free path is defined as a function of the Knudsen number and the location in the confined system, and it is always smaller than what would be obtained from a bounded system. For high Knudsen flows, gas molecules no longer have zero velocity near solid walls due to the strong slippage effect. In this regard, a proper boundary condition, combined bounce-back specular reflection (CBBSR), is discussed to capture the slippage effect. In kerogen, the interactions between organic pore walls and gas molecules are not negligible. We have incorporated the cohesive/adsorptive forces into the generalized LBM to understand the flow mechanisms with the 
adsorption effect in organic nanoscale porous media. A few numerical studies have been carried out based on this modified LBM to study the gas slippage and adsorption effects. We have simulated gas flow in a two-dimensional (2D) capillary tube with no adsorption to validate the present model, and the slippage effect has been observed. That simulation was followed by the simulation of gas flow in a 2D capillary tube with various amounts of adsorbed gas against different Knudsen numbers, and different velocity profiles have been observed accordingly. In addition to the commonly discussed dimensionless LBM results, we have also performed simulations with physical units in order to demonstrate the results in a more practical fashion. Methane gas flows, in four different capillary tubes with sizes ranging from $10 \mathrm{~nm}-80 \mathrm{~nm}$, have been simulated at the conditions of $T=300 \mathrm{~K}$ and $P=10 \mathrm{~atm}$. Finally, we have performed simulations on methane gas flows in two, 2D complex nano-porous structures with different porosities to investigate the slippage and adsorption effects. The gas permeability of these two porous media has been determined and the Klinkenberg constants have been obtained accordingly. Preliminary results demonstrate that the proposed LBM can effectively model and simulate natural gas flow in nano-pores, which can potentially improve the prediction of shale gas production performance and the estimation of stimulated reservoir volume.

\section{Methodology}

\subsection{Generalized lattice Boltzmann model}

The lattice Boltzmann method (LBM) has been widely used as an alternative and successful method for solving complicated physical, chemical, and fluid mechanics problems numerically (Qian et al., 1995; Premnath and Abraham, 2007; Premnath et al., 2009). The lattice Boltzmann equation (LBE) is a simplified Boltzmann equation in a discretized form, and it involves two fundamental terms: streaming and collision. The collision term in the Boltzmann equation originally involves a complex multivariable integral that is formidable to solve. In this regard, various models of the collision term have been developed in order to improve numerical stability and computational efficiency (Lallemand and Luo, 2000; Chen et al., 1992; Ginzburg, 2005; Geier et al., 2006; Premnath and Banerjee, 2009). One class of collision models is the generalized lattice Boltzmann method proposed by d'Humieres (1992) in which the LBE can be expressed as:

$$
f_{\alpha}\left(\vec{x}+\vec{e}_{\alpha} \delta_{t}, t+\delta_{t}\right)-f_{\alpha}(\vec{x}, t)=-\sum_{\beta} \Lambda_{\alpha \beta}\left[f_{\beta}(\vec{x}, t)-f_{\beta}^{e q}(\vec{x}, t)\right]+\delta_{t} \vec{F}_{\alpha} .
$$

In Equation (1), $f_{\alpha}$ is the density distribution function associated with gas molecules moving at the discrete velocity $\vec{e}_{\alpha}$, and it is illustrated in Figure 1 , where $\vec{e}_{\alpha}$ is given for a two-dimensional nine velocity model (D2Q9) as: 


$$
\vec{e}_{\alpha}= \begin{cases}(0,0) & \alpha=0 \\ c\left(\cos \left[(\alpha-1) \frac{\pi}{2}\right], \sin \left[(\alpha-1) \frac{\pi}{2}\right]\right) & \alpha=1,2,3,4 \\ \sqrt{2} c\left(\cos \left[(2 \alpha-1) \frac{\pi}{4}\right], \sin \left[(2 \alpha-1) \frac{\pi}{4}\right]\right) & \alpha=5,6,7,8\end{cases}
$$

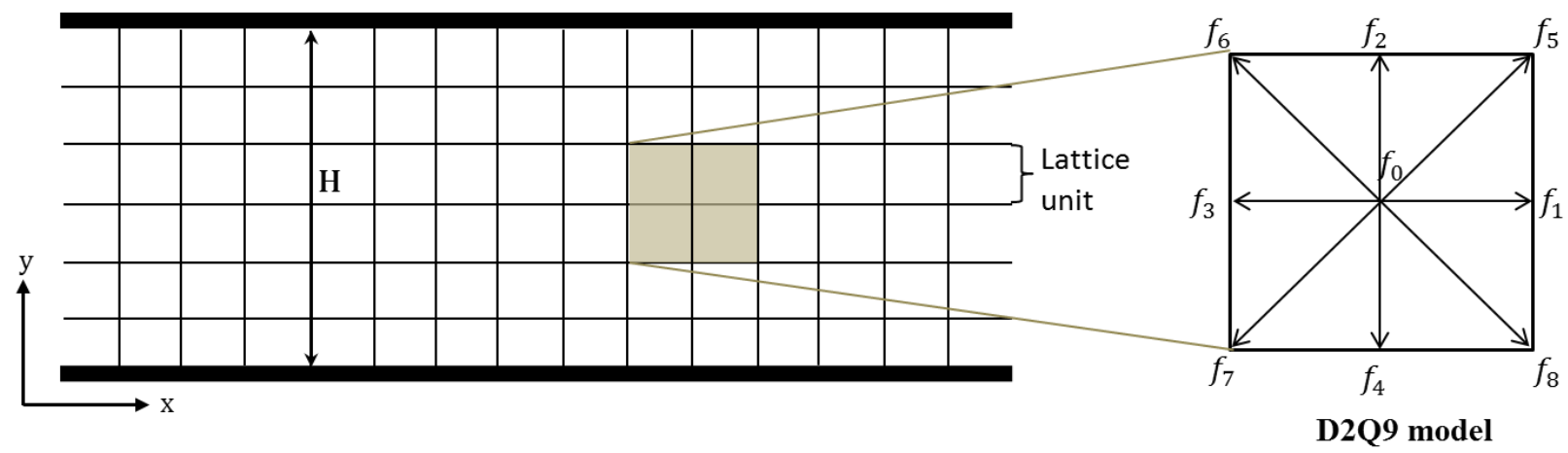

Figure 1: Two-dimensional lattice structure between two parallel plates and a typical D2Q9 lattice model

$c=\delta_{\mathrm{x}} / \delta_{\mathrm{t}}$ is the ratio of lattice spacing $\delta_{\mathrm{x}}$ and time step $\delta_{\mathrm{t}}$, and it is usually set to 1 . The left-hand side of Equation (1) is the streaming term that describes the propagations of the fluid particles on the lattices. The right-hand side of Equation (1) comprises the collision and source terms, in which the collision term describes the evolution of the distribution function to its equilibrium state and the source term $\delta_{t} \vec{F}_{\alpha}$ is a forcing experienced by molecules. $f_{\alpha}^{e q}$ is the equilibrium distribution function that can be calculated as:

$$
f_{\alpha}^{e q}=w_{\alpha} \rho\left[1+\frac{\vec{e}_{\alpha} \cdot \vec{u}}{c_{s}^{2}}+\frac{\left(\vec{e}_{\alpha} \cdot \vec{u}\right)^{2}}{2 c_{s}^{4}}+\frac{\vec{u} \cdot \vec{u}}{2 c_{s}^{2}}\right]
$$

which is an approximation of the Maxwell equilibrium function with $2^{\text {nd }}$ order accuracy based on Taylor expansion; $w_{\alpha}$ is the weighting factor that has $w_{0}=\frac{4}{9}, w_{i}=\frac{1}{9}(i=1,2,3,4)$, and $w_{i}=\frac{1}{36}(i=5,6,7,8) ; \rho$ is the fluid density; $\vec{u}$ is the fluid velocity; and $c_{S}=c / \sqrt{3}$ is the speed of sound.

In Equation (1), $\Lambda_{\alpha \beta}$ is the collision matrix that is $\boldsymbol{\Lambda}=\boldsymbol{M}^{-1} \boldsymbol{S} \boldsymbol{M}$ in a vector form, where $\boldsymbol{M}$ is a transformation matrix expressed in Equation (4), and $\boldsymbol{S}$ is the relaxation time matrix that is a diagonal matrix, $\operatorname{diag}(\boldsymbol{S})=\left(\tau_{\rho}, \tau_{e}, \tau_{\epsilon}, \tau_{j}, \tau_{q}, \tau_{j}, \tau_{q}, \tau_{s}, \tau_{s}\right)^{T}$, related to corresponding macroscopic variables. Lallemand and Luo (2000) proposed a column vector of macroscopic variables $m=$ $\left(\rho, e, \varepsilon, j_{x}, q_{x}, j_{y}, q_{y}, p_{x x}, p_{x y}\right)^{T}$, where $\rho$ is the fluid density, $\varepsilon$ is related to the square of the internal energy $e, j_{x}$ and $j_{y}$ are mass fluxes in $x$ and $y$ directions, respectively, $q_{x}$ and $q_{y}$ correspond to energy fluxes in both directions, and $p_{x x}$ and $p_{x y}$ correspond to the diagonal and off-diagonal components of the viscous stress tensor. For D2Q9 model, a $9 \times 9$ transformation matrix $\boldsymbol{M}$ is introduced to relate the 
moment vector $\vec{m}$ with the column vector, $\vec{f}=\left(f_{0}, f_{1}, f_{2}, f_{3}, f_{4}, f_{5}, f_{6}, f_{7}, f_{8}\right)$ (Lallemand and Luo, 2000), through

$$
\vec{m}=\left[\begin{array}{l}
\rho \\
e \\
\varepsilon \\
j_{x} \\
q_{x} \\
j_{y} \\
q_{y} \\
p_{x x} \\
p_{x y}
\end{array}\right]=\left[\begin{array}{ccccccccc}
1 & 1 & 1 & 1 & 1 & 1 & 1 & 1 & 1 \\
-4 & -1 & -1 & -1 & -1 & 2 & 2 & 2 & 2 \\
4 & 2 & 2 & 2 & 2 & 1 & 1 & 1 & 1 \\
0 & 1 & 0 & -1 & 0 & 1 & -1 & -1 & 1 \\
0 & -2 & 0 & 2 & 0 & 1 & -1 & -1 & 1 \\
0 & 0 & 1 & 0 & -1 & 1 & 1 & -1 & -1 \\
0 & 0 & -2 & 0 & 2 & 1 & 1 & -1 & -1 \\
0 & 1 & -1 & 1 & -1 & 0 & 0 & 0 & 0 \\
0 & 0 & 0 & 0 & 0 & 1 & -1 & 1 & -1
\end{array}\right]\left[\begin{array}{l}
f_{0} \\
f_{1} \\
f_{2} \\
f_{3} \\
f_{4} \\
f_{5} \\
f_{6} \\
f_{7} \\
f_{8}
\end{array}\right]=M \vec{f}
$$

The macroscopic quantities, the density of the fluid, the fluid velocity, and the fluid pressure can be obtained as, $\rho=\sum_{\alpha} f_{\alpha}, \vec{u}=\sum_{\alpha} \vec{e}_{\alpha} f_{\alpha}+\frac{\delta_{t}}{2} \vec{F}$, and $P=c_{s}^{2} \rho$, respectively.

One immediate advantage of the GLBE model is that macroscopic variables of interest can be readily obtained by simply performing the matrix multiplication $\boldsymbol{M} \vec{f}$ if $\vec{f}$ is known. However, the BGK model allows all $f_{\alpha}$ to be relaxed at the same rate, which limits the possible types of evolution of the macroscopic quantities. Different moments should be relaxed at different relaxation times, which allows for implementing complex physics more naturally. If the relaxation time matrix $\boldsymbol{S}$ is set to $\boldsymbol{S} \boldsymbol{I}$, the GLBE model returns to the BGK model. The selection of these relaxation times depends on the type of flow and the boundary conditions, which will be discussed in Sections $\mathbf{2 . 2}$ and $\mathbf{2 . 3}$.

\subsection{Effective relaxation times.}

Gas flows in micro/nano systems have large Knudsen numbers $\left(K_{n}\right)$ because the mean free path of gas molecules has the same order of magnitude as the characteristic length of the flow region. It is wellaccepted that the Boltzmann equation can be used to model gas flows ranging from the continuum regime $K_{n}<0.001$ to the free molecular regime $K_{n}>10$ (Guo et al., 2006; Shan et al., 2006). Since the standard lattice Boltzmann equation (LBE) is a much simplified version of the Boltzmann equation, most of the available LBE models are inadequate for flows in the transition regime $K_{n}>0.1$. The failure of the standard LBE for high Knudsen flows can be understood as its insufficient ability to capture the Knudsen layers near solid surfaces. The Knudsen layer is always formed when gas flows over a solid surface with a thickness of $O(\lambda)$, as illustrated in Figure 2. Within the Knudsen layer, since the collisions are not sufficient to reach the quasi-thermodynamic-equilibrium, the N-S equations fail to work. The standard LBE model is an approximation to the Boltzmann equations only in terms of the accuracy level of the N-S 
equations, so it also fails to describe the flow within the Knudsen layer. One approach for successfully capturing the Knudsen layer is to make use of an effective relaxation time.

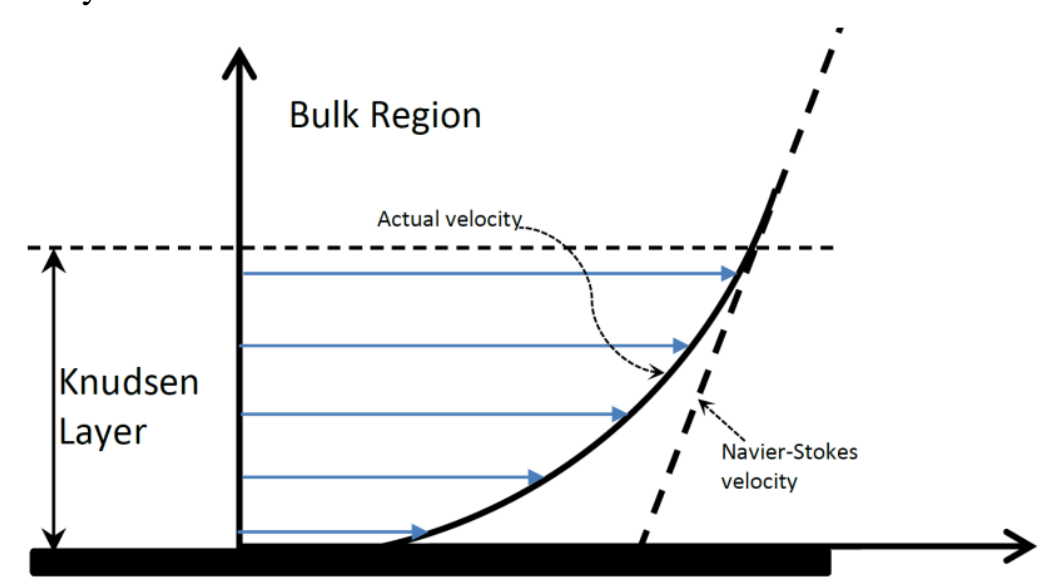

Figure 2: Illustration of the Knudsen layer

Due to frequent encounters with solid walls, the mean free path of gas molecules within the Knudsen layer becomes smaller than it is in the bulk region. In addition, the mean free path in a bounded system is always smaller than it is in an unbounded system. Therefore, we need an effective mean free path that depends on both the Knudsen number and the location of the gas molecules in the flow system. The effective mean free path can be calculated as:

$$
\lambda_{e}=\lambda_{\infty} \Psi\left(K_{n}, \vec{x}\right),
$$

where $\lambda_{\infty}$ is the mean free path in an unbounded system defined as:

$$
\lambda_{\infty}=\frac{k_{B} T}{\sqrt{2} \pi P d^{2}}
$$

where $k_{B}$ is the Boltzmann constant, $T$ is the system temperature, $P$ is the fluid pressure, and $d$ is the diameter of the gas molecule. The exact expression of $\Psi$ can be derived through the probability distribution function of the free path of the gas molecules (Stops, 1969). For simplicity, a gas bounded between two parallel plates is taken as an example, where the plates are located at $z=0$ and $z=H$. $\Psi$ between parallel plates is:

$$
\Psi(z)=\frac{1}{2}\left[\varnothing\left(\frac{z}{\lambda_{\infty}}\right)+\emptyset\left(\frac{H-z}{\lambda_{\infty}}\right)\right]
$$

where the function $\emptyset$ is defined as:

$$
\varnothing(y)=1+(y-1) e^{-y}-y^{2} E_{i}(y)
$$

where $E_{i}(y)$ is the exponential integral function. As shown in Figure 3, the effective mean free path is plotted against the location between the two parallel plates for various Knudsen numbers. It is seen that 
$\lambda_{e}$ is close to $\lambda_{\infty}$ in the central region for $K_{n}=0.01$, but it becomes smaller when it is closer to the walls. As the Knudsen number increases, the confinement effects become more significant. At $K_{n}=10, \lambda_{e}$ becomes less than $10 \%$ of $\lambda_{\infty}$.

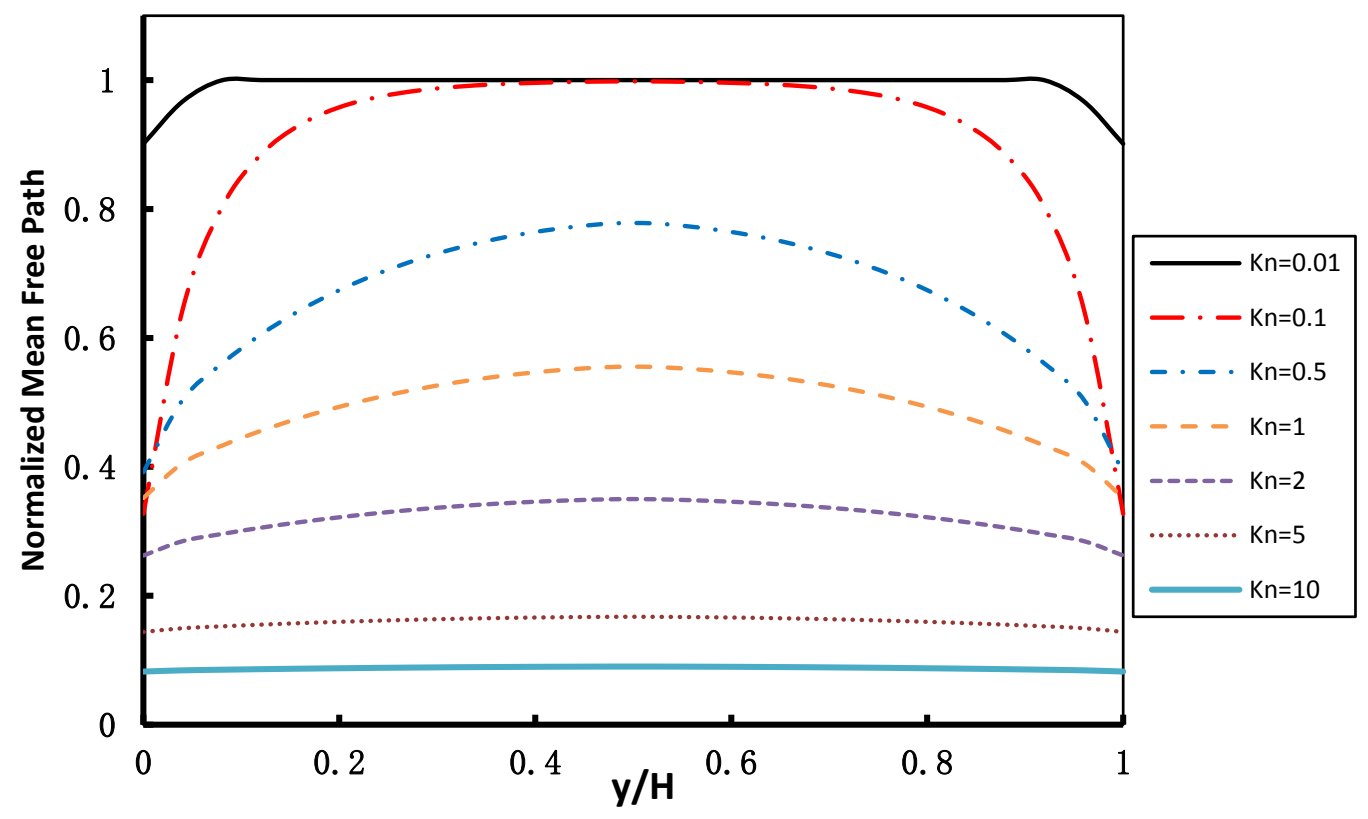

Figure 3: Effective mean free path at different Knudsen numbers

As mentioned in Section 2.1, the generalized LBE has multiple relaxation times, i.e. $\operatorname{diag}(\boldsymbol{S})=$ $\left(\tau_{\rho}, \tau_{e}, \tau_{\epsilon}, \tau_{j}, \tau_{q}, \tau_{j}, \tau_{q}, \tau_{s}, \tau_{s}\right)^{T}$. Since the macroscopic variables are driven to their equilibrium states at the corresponding relaxation times, the selection of these relaxation times significantly affects the macroscopic variables. Failure to choose the correct relaxation times can cause numerical instability and inaccuracy (Lallemand and Luo, 2000). $\tau_{\rho}$ and $\tau_{j}$ are related to the conserved quantities, mass and momentum, respectively. Therefore, while different values don't change the corresponding quantities, they do affect the rate of relaxations to their equilibrium states. $\tau_{e}$ and $\tau_{\epsilon}$ are the relaxation times related to macroscopic quantity, internal energy, so they are unimportant for an isothermal flow system, namely natural gas flow in an isothermal or nearly isothermal reservoir condition. The remaining relaxation time $\tau_{q}$ is a constant that can be determined from the non-slip boundary condition. However, when slip boundary behavior appears on a solid boundary, it has been found to have a significant influence on the accuracy of the slip velocity (Guo et al., 2008; Ginzburg and d'Humieres, 2003). For natural gas flow in a confined pore, $K_{n}$ is finite and the Knudsen layer occupies a large portion of the flow domain, which results in a pronounced slip boundary velocity, so $\tau_{q}$ becomes important for describing the slip phenomenal on the pore wall. $\tau_{q}$ is given in (Guo et al., 2008; Li et al., 2011), which is a function of the 
$K_{n}$ number and the accommodation coefficient of the solid surface $\sigma . \tau_{q}=0.5+\frac{3+4 \pi \bar{\tau}_{s}^{2} A_{1}}{16 \bar{\tau}_{s}}$, where $A_{1}=$ $\frac{1}{\pi}+\frac{A_{2}^{2}}{2}, \quad A_{2}=\frac{2-\sigma}{\sigma}(1-0.1817 \sigma), \bar{\tau}_{s}=\tau_{s}(0)-0.5$. As pointed out in (Fathi and Akkutlu 2013), the accommodation coefficient is free parameter that relates to diffusive reflection of solid surface, which influences the slip velocity significantly. Therefore, the impact of this accommodation coefficient will be investigated in the simulation results section. $\tau_{s}$ drives the macroscopic variable, viscous stress tensor, to its equilibrium state, and it is related to the effective mean free path as:

$$
\tau_{s}=\frac{1}{2}+\sqrt{\frac{2}{\pi R T}} \frac{\lambda_{e}}{\delta_{x}}
$$

where $R$ is the gas constant.

\subsection{Gas-solid boundary conditions.}

Generally in fluid dynamics, initial and boundary conditions are given in terms of macroscopic variables, such as pressure, velocity, temperature, etc. However, the basic variable for the LBM is distribution function. Although it is easy to obtain the macroscopic variables based on distribution functions, it is rather difficult to determine the distribution functions from the macroscopic quantities. Therefore, it is important to define initial and boundary distribution functions properly from the given macroscopic conditions for LBM. The initial condition does not contribute much to the computations if the flow is at a steady or quasi-steady state (Yu et al., 2005). In this work, most of the tests are for steady flows or nearly steady flows, so $f_{\alpha}=f_{\alpha}^{e q}, \rho_{0}=$ costant, $u_{x}=u_{y}=0$, and $p=\rho c_{s}^{2}$ were set for the initial conditions. Two types of boundary conditions are widely used in LBM simulations: the bounceback (BB) and the specular-reflection. The bounce-back boundary condition is used for treating non-slip boundary velocity in a continuum flow regime, namely $K_{n}<0.001$. However, this boundary condition is not suitable for natural gas flow in nanoscale pores where the Knudsen layer is not negligible. As we discussed previously, the gas slippage effect on solid boundaries occurs quite often. Some schemes have been proposed for the purpose of the slip boundary condition, such as the combined bounce-back specular-reflection (CBBSR) (Sbragaglia and Succi, 2005) and the discrete Maxwell's diffuse-reflection (DMDR) (Tang et al., 2005a). In this work, we used the CBBSR scheme due to its simplicity compared with the DMDR scheme,

$$
f=r f_{B B}+(1-r) f_{S R}
$$

where $0 \leq r \leq 1$ is the portion of the bounce-back part in the combination, $f_{B B}$ is the distribution function of bounce-back part, and $f_{S R}$ is the distribution function of specular reflection part. Taking the 
bottom solid boundary in Figure 1 as an example, $f_{B B}$ is calculated as $f_{2}=f_{4}, f_{5}=f_{7}$, and $f_{6}=f_{8}$, and $f_{S R}$ is calculated as $f_{2}=f_{4}, f_{5}=f_{8}$, and $f_{6}=f_{7}$. In the CBBSR scheme, the portion $r$ determines the slippage intensity on the solid boundaries (Zhang et al., 2014). In most of the previous studies, $r$ was chosen as a constant rather freely, which causes the velocities on the boundaries to greatly depend on this undetermined value. Recently, it was shown that $r$ mainly depends on the $K_{n}$ number and the surface properties (Guo et al., 2008). In this work, we used the proposed model for $r$ found in (Guo et al., 2008), which is $r=\left[1+\sqrt{\frac{\pi}{6}} A_{2}+\frac{\tau_{S}^{\prime}(0) \delta_{x}}{8 \bar{\tau}_{s}^{2}}\right]^{-1}$.

\subsection{Adsorption effects on organic surfaces.}

In shale formations, it has been shown that a large amount of natural gas is adsorbed on kerogen nanopore walls (Kang et al., 2011). The interaction between adsorbed gas and free gas may have an important impact on gas flow behavior. To model this interaction, we need to introduce a long-range interaction force between the fluid particles. Shan and Chen (1993) proposed a pseudo-potential LBM model in which the fluid interactions are modeled by an interparticle potential. The cohesive force between the fluid particles is obtained from the interparticle potential, and the molecular interactions cause phase segregation that is modeled by this cohesive force. Since the cutoff radius of the Lennard-Jones potential is normally 3 times of the molecule's size and the size of the lattice in our simulations is at least 3 times larger than the size of molecules, the interactions among particles at the neighboring lattices are sufficient. The cohesive force is given as (Shan and Chen, 1993):

$$
\vec{F}(\vec{x}, t)=G \Phi(\vec{x}, t) \sum_{\alpha} w_{\alpha} \Phi\left(\vec{x}+\vec{e}_{\alpha} \delta_{t}, t\right) \vec{e}_{\alpha},
$$

where $G$ is the intermolecular strength, negative for attractive and positive for repulsive and $\Phi$ is the

monotonically increasing pseudo-potential defined as $\Phi(\vec{x}, t)=\rho_{0} \exp \left(1.0-\frac{\rho(\vec{x}, t)}{\rho_{0}}\right)$, where $\rho_{0}$ is a constant. Other forms of the pseudo-potential can also apply (Martys and Chen, 1996; Qian et al., 1995). Note that $G$ is a function of temperature and can be determined through the graph of pressure/density for a specific gas (Sukop and Or, 2004). Introduction of the interaction forces between particles in LBM leads to a non-ideal equation of state (EOS) that can be used to model the interphase dynamics between phases (He and Doolen, 2002):

$$
P=\rho R T+\frac{G R T}{2} \Phi^{2}(\rho)
$$


The van der Waals EOS for a pure methane system at temperature $T=300 \mathrm{~K}$ is illustrated as the solid line in Figure 4. The coefficients $\rho_{0}$ and $G$ are chosen to be 3.0 and -0.1 by matching the pressuredensity curve of the van der Waals EOS. $G$ can be different for different temperatures and type of gases.

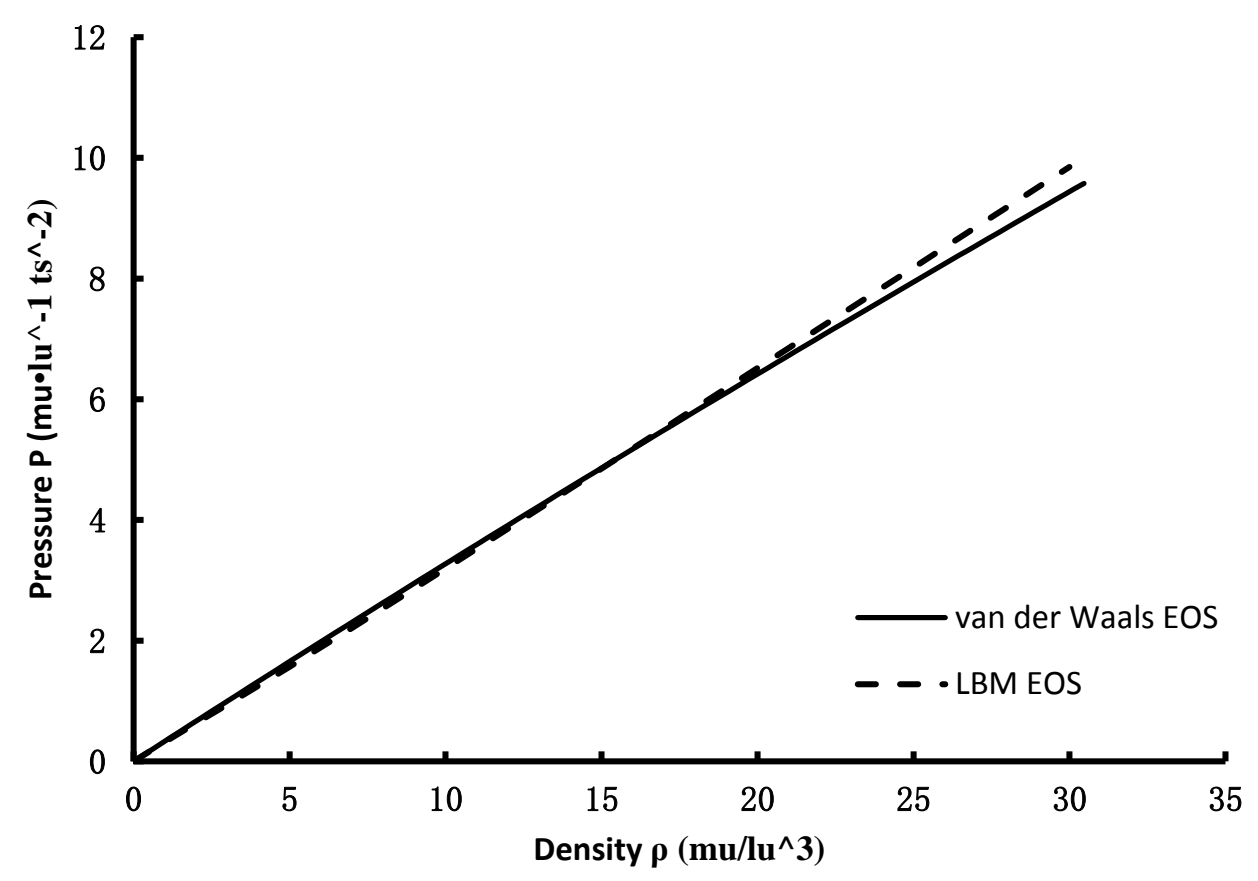

Figure 4: Methane pressure/density diagram

To describe the interactions between fluid molecules and solid pore walls, we use an adsorptive force introduced by (Sukop and Or, 2004):

$$
\vec{F}_{a d s}(\vec{x}, t)=G_{a d s} \Phi(\vec{x}, t) \sum_{\alpha} w_{\alpha} s\left(\vec{x}+\vec{e}_{\alpha} \delta_{t}, t\right) \vec{e}_{\alpha}
$$

where $G_{a d s}$ is the adsorptive strength, negative for wetting and positive for non-wetting; $s$ is a switch function, which tells $s\left(\vec{x}+\vec{e}_{\alpha} \delta_{t}, t\right)=1$ if $\vec{x}+\vec{e}_{\alpha} d t$ is solid, $s\left(\vec{x}+\vec{e}_{\alpha} \delta_{t}, t\right)=0$ if otherwise. The meaning of this equation is that adsorptive forces only exist if the gas molecules are at the solid nearest lattice nodes. $G_{a d s}$ was set constant in most of the previous papers (Sukop and Or, 2004; Fathi and Akkutlu, 2013; Sukop and Thorne Jr., 2006). In this work, we chose $G_{a d s}=\rho_{\max } \exp \left(1.0-\frac{\rho(\vec{x}, t)}{\rho_{\max }}\right)$, where $\rho_{\max }$ is the maximum capacity of the adsorbed gas.

\section{Numerical Results}

\subsection{Slippage effect of gaseous micro-scale flows.}


The slippage effect can significantly enhance gas transport in micro-scale pores, which is largely controlled by the accommodation coefficient $\sigma$ in the present model. A proper choice of the accommodation coefficient is significantly important to our simulation results. This coefficient indicates the diffusive reflection of gas molecules on solid boundaries, i.e. $\sigma=1.0$ represents fully diffusive reflection. In order to investigate the impact of the accommodation coefficient, we have simulated a simple 2D micro/nano channel flow with the width of 50 lattice units (lu) for $K_{n}=0.132$. The flow was driven by a constant body force $\boldsymbol{a}$ and its velocity profile has a parabolic-shape. The initial condition is $f_{\alpha}=f_{\alpha}^{e q}, \rho_{0}=3.0, u_{x}=u_{y}=0$. The periodic inlet/outlet condition and the CBBSR boundary condition were used in this simulation. The cohesive and adsorptive forces were ignored $(G=$ $0 \& G_{a d s}=0$ ). The velocities are normalized by the maximum velocities at the center. In this simulation, different values of the accommodation coefficient $\sigma$ have been tested and compared with the Molecular Dynamics results (Suga et al., 2010). As shown in Figure 5, compared with the results of Molecular Dynamics for an identical problem, $\sigma=1.0$ seems to be a very reasonable choice, while the results with smaller $\sigma$ give overestimation on boundaries. One should notice that $\sigma=1$ is a common assumption in many published papers (Ohwada et al., 1989; Guo et al., 2008; Li et al., 2011), which is that gas molecules are reflected diffusely on the boundaries. Therefore, we set $\sigma=1$ in our latter simulations. Using the same simulation condition, we have demonstrated the slippage effect of micro-scale gas flow for different Knudsen numbers in Figure 6. It can be clearly observed that the slippage effect becomes more pronounced as the Knudsen number increases. Moreover, the Molecular Dynamics results (Suga et al., 2010) validate the present model well. 


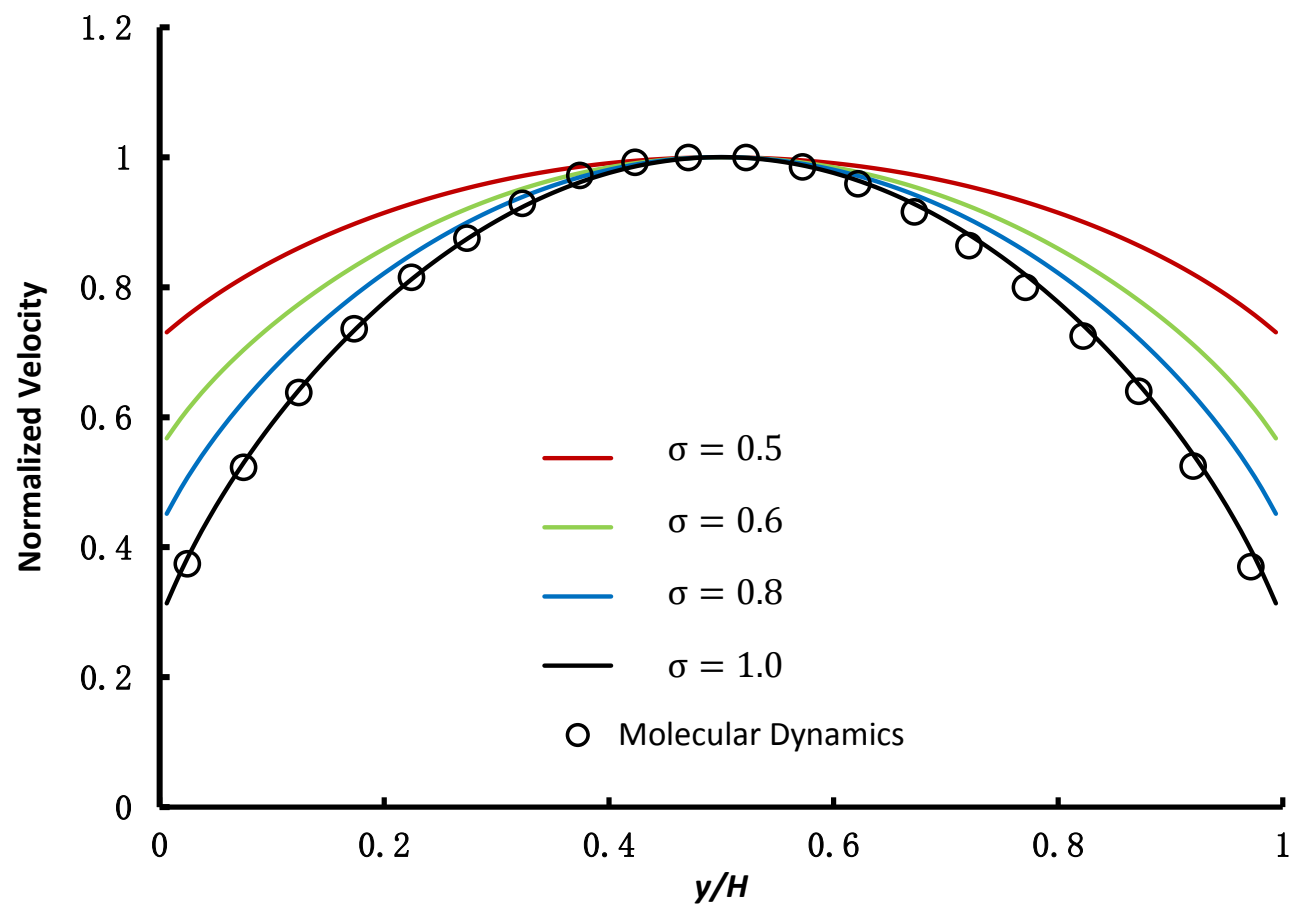

Figure 5: Impact of accommodation coefficients

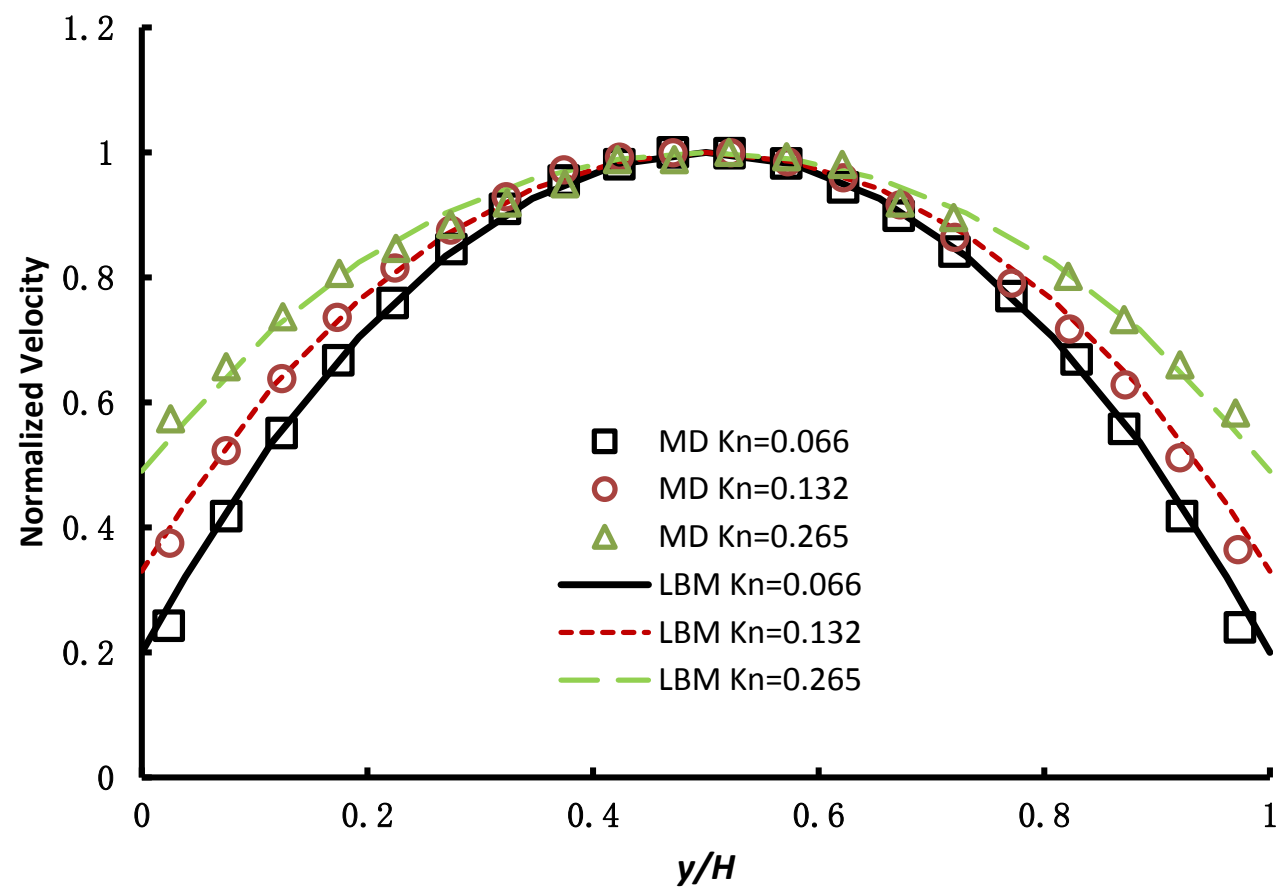

Figure 6: Comparison of velocities at different Knudsen numbers

The mass fluxes in the channel normalized by $\rho \boldsymbol{a} H^{2} / \sqrt{2 R T}$ were calculated to further validate the present model, shown in Figure 7. As seen, the present model agrees well with the solution of the linearized Boltzmann equation (Ohwada et al., 1989) up to $K_{n}=1.0$. In typical shale formations, a possible maximum value of the Knudsen number is less than 1.0 for methane molecules with a diameter 
of $0.37 \mathrm{~nm}$, a pore size ranging from $2 \mathrm{~nm}-1 \mu \mathrm{m}$, temperature ranging from $100 \mathrm{~F}-500 \mathrm{~F}$ and pressure ranging from 1000 psi- 5000 psi, which means the current model is suitable for such high Knudsen flows.

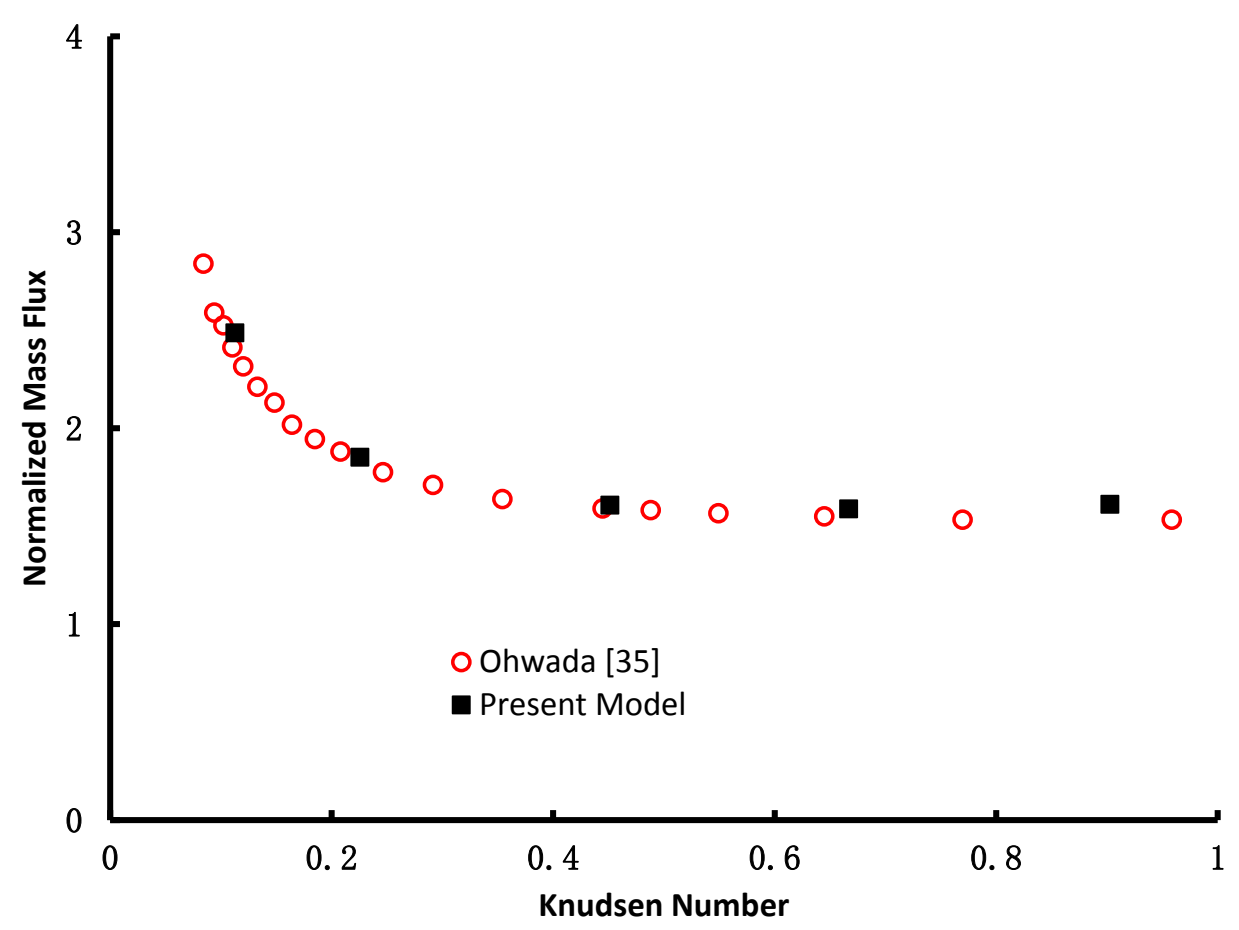

Figure 7: Comparison of mass fluxes at different Knudsen numbers

\subsection{Adsorption effect of gas flows in organic tube(s).}

Organic-rich shale formations contain numerous organic nano-pores and the gas flows in those small pores may be highly influenced by the slippage and adsorption effects. In this regard, a single organic nanotube has been simulated at different Knudsen numbers. Periodic inlet/out condition, CBBSR boundary condition, a constant body force $\boldsymbol{a}$, and the same adsorptive/cohesive force $\left(G_{a d s}=-0.1, G=\right.$ -0.1 ) have been applied to different Knudsen flows. The velocity profile is normalized by its maximum velocity at the central region. As shown in Figure 8a, the velocity profile changes significantly at different Knudsen numbers. At $K_{n}=0.01 \& 0.1$, the profile has a parabolic shape. As the Knudsen number increases, the boundary effect due to adsorption becomes more important. At $K_{n}=0.5$, the velocity on the boundary is no longer smaller than the velocity in the bulk region, which means that for a larger Knudsen number, namely extremely small pores, i.e., $H<<1 \mu \mathrm{m}$, the transport of the adsorbed gas can contribute a large portion of the gas transport. In addition, as shown in Figure 8b, the density profiles of the different Knudsen numbers are similar. 


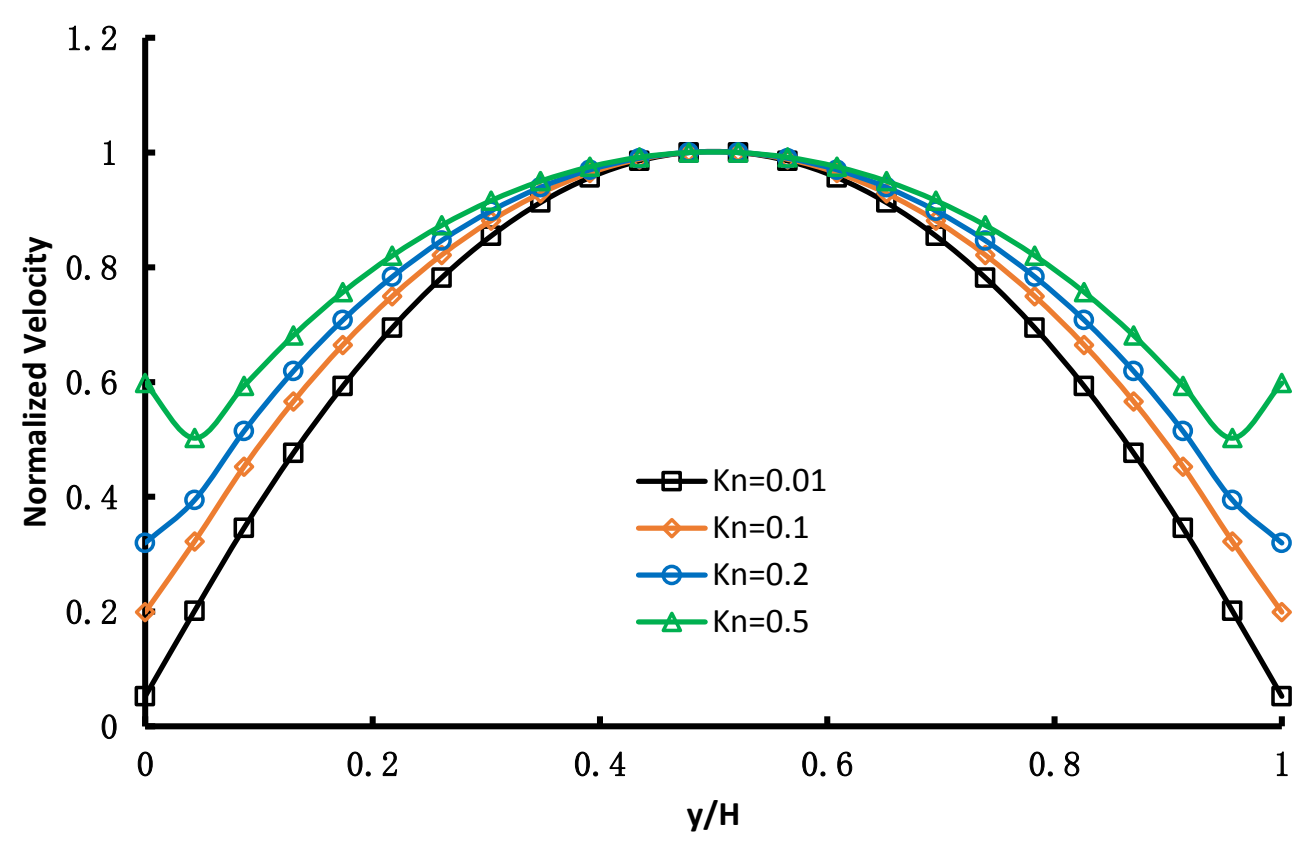

$8 \mathrm{a}$

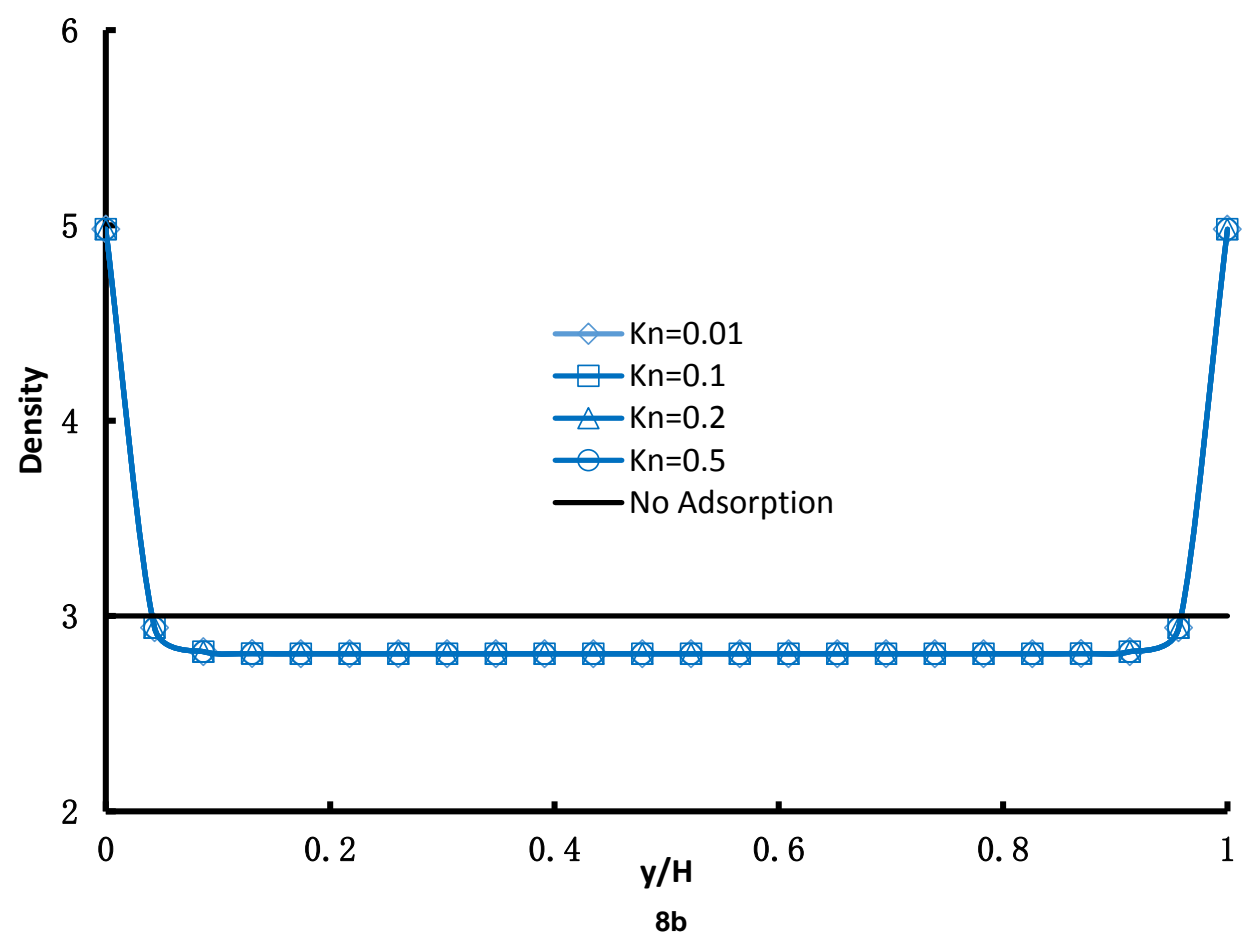

Figure 8: Velocity and density profiles at different Knudsen numbers

As seen in Figure 9, methane gas flows in four capillary tubes with different sizes, $10 \mathrm{~nm}, 20 \mathrm{~nm}, 40 \mathrm{~nm}$, and $80 \mathrm{~nm}$, respectively, have been simulated. For an isothermal system at $T=300 \mathrm{~K}$ and $P=10 \mathrm{~atm}$, the gravitational acceleration $\boldsymbol{a}=(0,9.8) \mathrm{m} / \mathrm{s}^{2}$ is added to the methane molecules, and the periodic inlet/outlet condition and the CBBSR boundary condition are used. The 
intermolecular strength $G$ is chosen to be -0.1 by the match methane pressure/density diagram at $300 \mathrm{~K}$, and the adsorptive strength $G_{a d s}=-3\left[1-\exp \left(-\frac{\rho}{3}\right)\right]$. The diameter of a methane molecule is approximately $0.37 \mathrm{~nm}$ and its mean free path at this simulation condition is $6.72 \mathrm{~nm}$. The dash lines are the simulation results for gas flows without the adsorption effect, and the solid lines are the results for flows with adsorptions. The average velocities for the different tube sizes are given in Table 1. It can be observed that the velocity reduction due to adsorption is increased as the size of tube decreases. In another word, when the tube size is large enough or the Knudsen number is very small, the adsorption effect can become negligible.

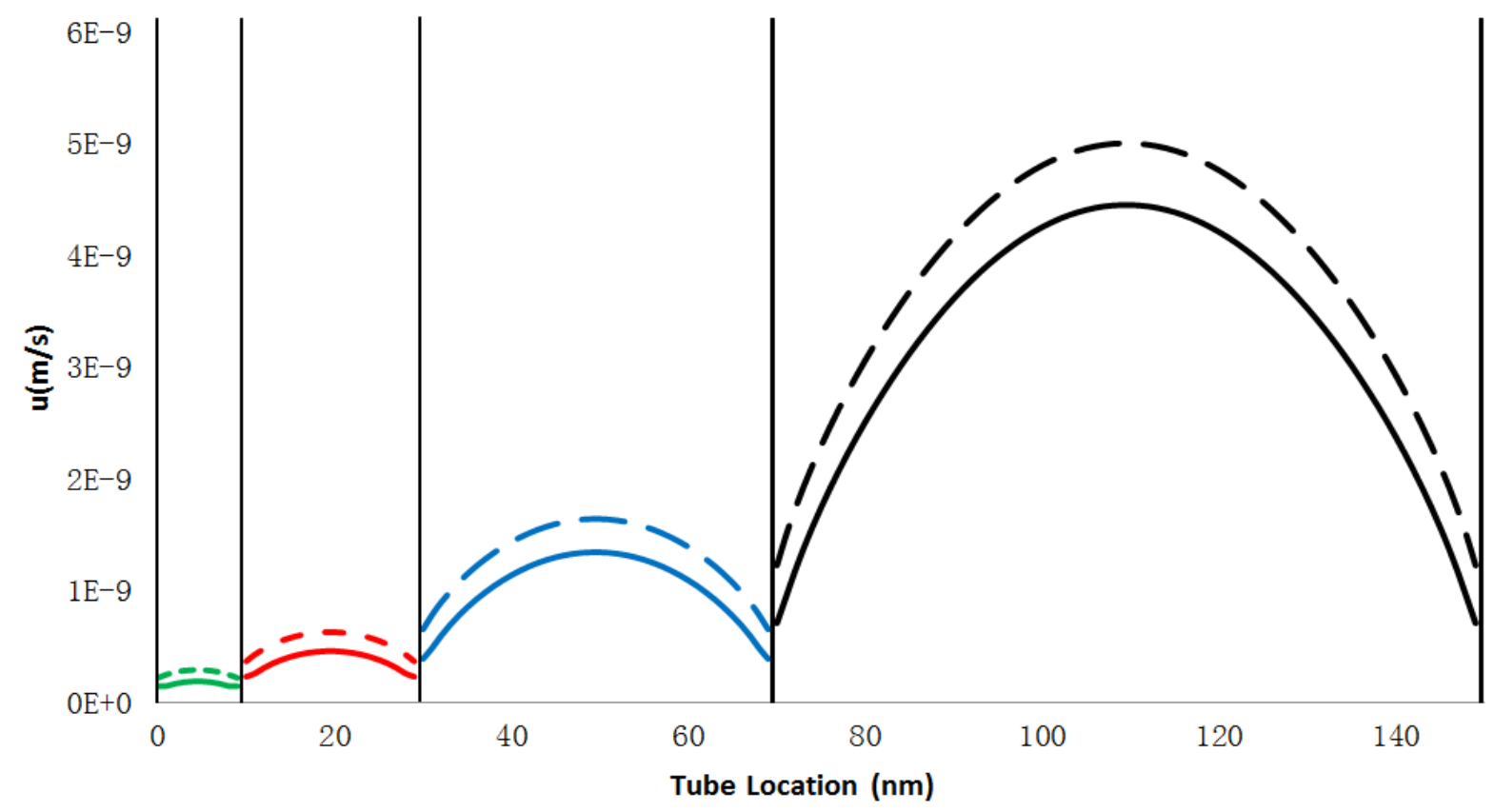

Figure 9: Velocity profiles for different organic nano-tube sizes

\begin{tabular}{|c|c|c|c|c|}
\hline Tube size & $10 \mathrm{~nm}$ & $20 \mathrm{~nm}$ & $40 \mathrm{~nm}$ & $80 \mathrm{~nm}$ \\
\hline$K_{n}$ & 0.672 & 0.336 & 0.168 & 0.084 \\
\hline$u(n m / s)$ no adsorption & 0.261 & 0.537 & 1.320 & 3.793 \\
\hline$u(n m / s)$ with adsorption & 0.164 & 0.370 & 1.024 & 3.241 \\
\hline Velocity reduction \% & $37.2 \%$ & $31.1 \%$ & $22.4 \%$ & $14.6 \%$ \\
\hline
\end{tabular}

Table 1: Averaged velocities for different sizes of organic nano-tubes

As shown in Figure 10, the sensitivity to the adsorption amount on the pore walls has been investigated on a $2 \mathrm{D}$ organic tube with the length of $200 \mathrm{~nm}$ and the width of $80 \mathrm{~nm} . \mathrm{T}=300 \mathrm{~K}, P=$ 
$10 \mathrm{~atm}, \boldsymbol{a}=(0,9.8) \mathrm{m} / \mathrm{s}^{2}$, and the periodic inlet/out condition and the CBBSR boundary condition are used for this simulation. The Knudsen number in this case is 0.084 . Assuming that the same total amount of methane molecules $\left(1.0 \times 10^{-10} \mathrm{gram}\right)$ is maintained in the tube, the amounts of adsorbed and free molecules varied. It is observed that the average velocity in the tube decreases as the maximum capacity of the monolayer adsorption on the organic surface increases. When no adsorption occurs on the pore walls, the velocity is higher than the velocity when adsorption occurs. This adsorption effect becomes more significant as more adsorbed gas arises. Average velocities for different amounts of adsorbed gas are shown in Table 2. The average velocity for $\rho_{\text {free }}=\rho_{\text {ads }}$ is about $30 \%$ higher than the average velocity for $\rho_{\text {free }}=3.08 \rho_{a d s}$; thus, it can be concluded that the adsorption effect can significantly influence the prediction of flow rates in shale formations.

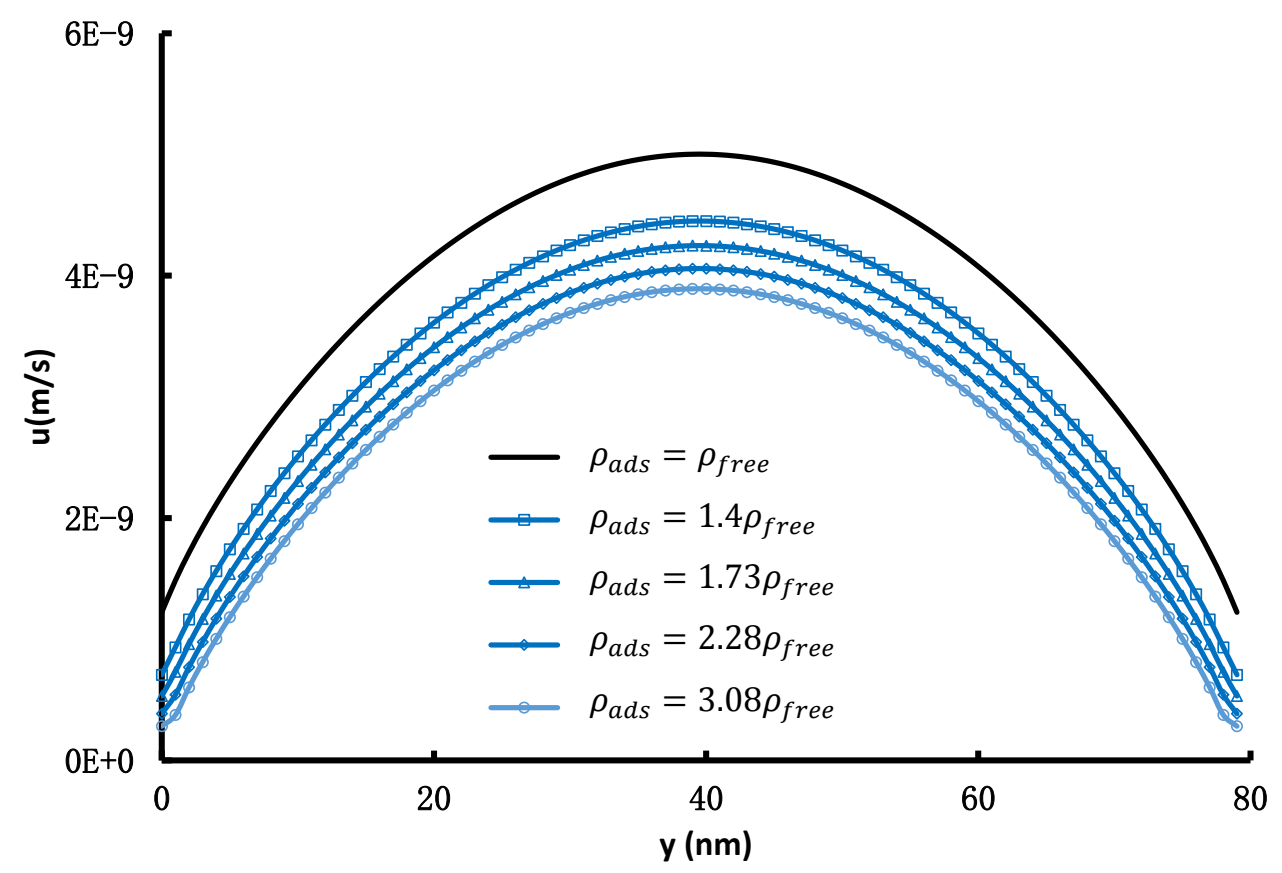

Figure 10: Velocity profiles for different adsorption amounts

\begin{tabular}{|c|c|c|c|c|c|}
\hline$\frac{\rho_{\text {ads }}}{\rho_{\text {free }}}$ & 1 & 1.4 & 1.73 & 2.28 & 3.08 \\
\hline$u(n m / s)$ & 3.7926 & 3.2415 & 3.0397 & 2.8509 & 2.6855 \\
\hline
\end{tabular}

Table 2: Average velocity for different adsorption amounts

\subsection{Investigation of effective gas permeability on complex porous structures.}

Matrix permeability of shale is an important property that controls long-term performance of gas wells, and it is dependent of multiple conditions, such as pore pressure, temperature, organic content, etc. Determination of shale permeability remains challenging both experimentally and theoretically 
(Ghanizadeh et al., 2015). Experimentally, there are several different methods for measuring shale permeability, i.e. steady-state (Zamirian et al., 2014) pressure pulse-decay (Heller et al., 2014) and crushed sample (Fathi et al., 2012) measurements. Each approach has its limitations and gives very different values, sometimes several orders of magnitude (Ghanizadeh et al., 2015). Many theoretical works based on Klinkenberg equation have been proposed in order to effectively describe gas permeability in low-permeability porous media (Moghadam and Chalaturnyk, 2014; Fathi et al., 2012; Ziarami and Aguilera, 2012; Zhu et al., 2007). According to the Klinkenberg theory (Klinkenberg, 1941), gas permeability follows:

$$
k_{g}=k_{\infty}\left(1+\frac{b}{\bar{p}}\right)
$$

where $k_{\infty}$ is the permeability at the infinite pressure or the liquid permeability; $b$ is a slippage factor; and $\bar{p}$ is the mean pressure of the porous media. In tight and shale gas reservoirs, it has been investigated that the gas permeability has higher-order dependency with the mean pressure (Moghadam and Chalaturnyk, 2014; Fathi et al., 2012; Ziarami et al., 2012; Tang et al., 2005b; Zhu et al., 2007). The general higherorder form or the Klinkenberg-corrected permeability can be written as:

$$
k_{g}=k_{\infty}\left(1+\frac{b}{\bar{p}}+\frac{a}{\bar{p}^{2}}\right)
$$

where $a$ is a constant that depends on gas properties and pore geometry. The values of $a$ and $b$ have been found differently in (Moghadam and Chalaturnyk, 2014; Fathi et al., 2012; Ziarami and Aguilera, 2012; Tang et al., 2005b; Zhu et al., 2007). a has been reported to be a negative value in (Moghadam and Chalaturnyk, 2014) and a positive value instead in (Fathi et al., 2012; Tang et al., 2005). The true relationship between gas permeability and the mean pressure still remains unclear, and it could be very different depending on testing methodology and shale's characteristics.

Another way to estimate shale gas permeability is to make use of simulation tools. In this regard, Gas flows through two porous media $(150 \mathrm{~nm} \times 150 \mathrm{~nm})$ with porosity $\epsilon=0.6$ and 0.3 , shown in Figure $11.1 \mathrm{a}$ and $11.1 \mathrm{~b}$, respectively, have been simulated to investigate the gas permeability of methane. The characteristic lengths of the two media are $30 \mathrm{~nm}(\epsilon=0.6)$ and $15 \mathrm{~nm}(\epsilon=0.3)$. The outlet pressure was fixed at $10 \mathrm{~atm}$ and the inlet pressures varied. The temperature for the two porous media was $500 \mathrm{~K}$, in which the chosen intermolecular strength was $G=-0.01$ by matching the van der Waals EOS. The adsorptive strengths were set at $G_{a d s}=0,-0.01$ and -0.02 , respectively, to investigate the adsorption effects on the permeability. Figure $11.2 \mathrm{a}$ and $11.2 \mathrm{~b}$ show the streamlines of gas flow for $G_{a d s}=0$, while 11.3a and $11.3 \mathrm{~b}$ show the streamlines of gas flow for $G_{a d s}=-0.01$. For complex structures, the effective relaxation time $\tau_{s}$, discussed earlier in Section $\mathbf{2 . 2}$, becomes inapplicable since it was limited to straight 
walls. In this regard, we used $\tau_{s}=0.5+\sqrt{6 / \pi} N K_{n} /\left(1+\theta K_{n}\right)$ as found in (Li et al., 2011), where $\theta=$ 2 and $N$ is the number of the lattices at the characteristic length. The average gas permeability is evaluated from pressure and velocity data based on a non-Klinkenberg gas flow solution of compressible gas (Scheidrgger, 1972) as follows:

$$
k_{g}=\frac{2 \mu L q_{o} P_{o}}{P_{i}^{2}-P_{o}^{2}}
$$

where $q_{o}$ is the outlet velocity and $P_{i}$ and $P_{o}$ are the inlet and outlet pressure, respectively. The permeability is a function of the reciprocal mean pressure of the porous media, as shown in Figure 12. Gas permeability has micro-Darcy units and decreases almost linearly when pressure is increased. The non-linearity effect is observed for the pore structure with $\epsilon=0.3$ due to the second-order term in Eq. (14). The permeability for $\epsilon=0.3$ media is significantly smaller than the permeability for $\epsilon=0.6$ media. It is also observed that the permeability is reduced by enhancing the adsorptive strength. Klinkenberg constants $k_{\infty}$ and $b$ have also been evaluated. If porosity is smaller, i.e., $\epsilon<10 \%$, and pore connectivity is poor, gas permeability could drop to nano-Darcy units, which is more consistent with real 3D kerogen porous media.

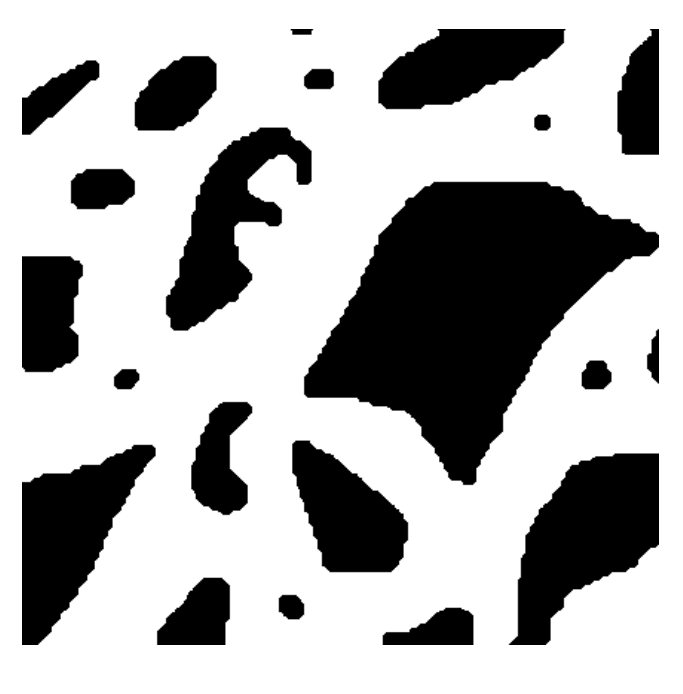

11.1a

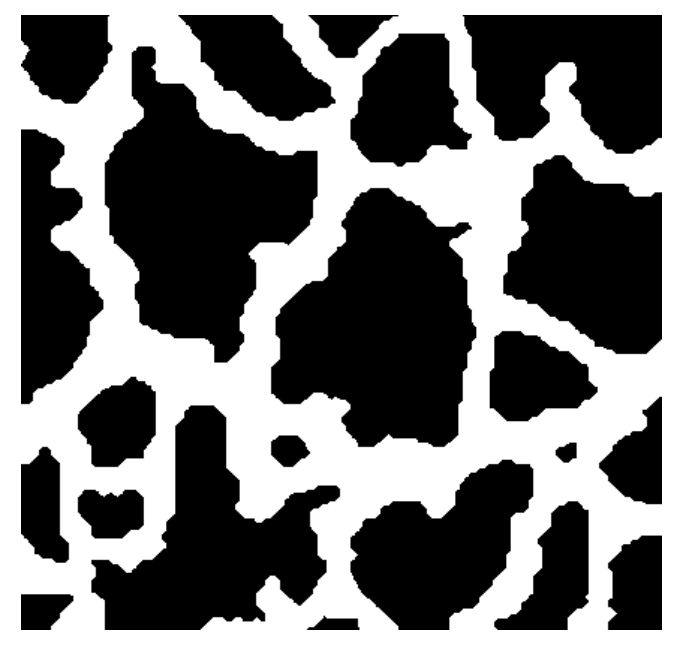

$11.1 b$ 

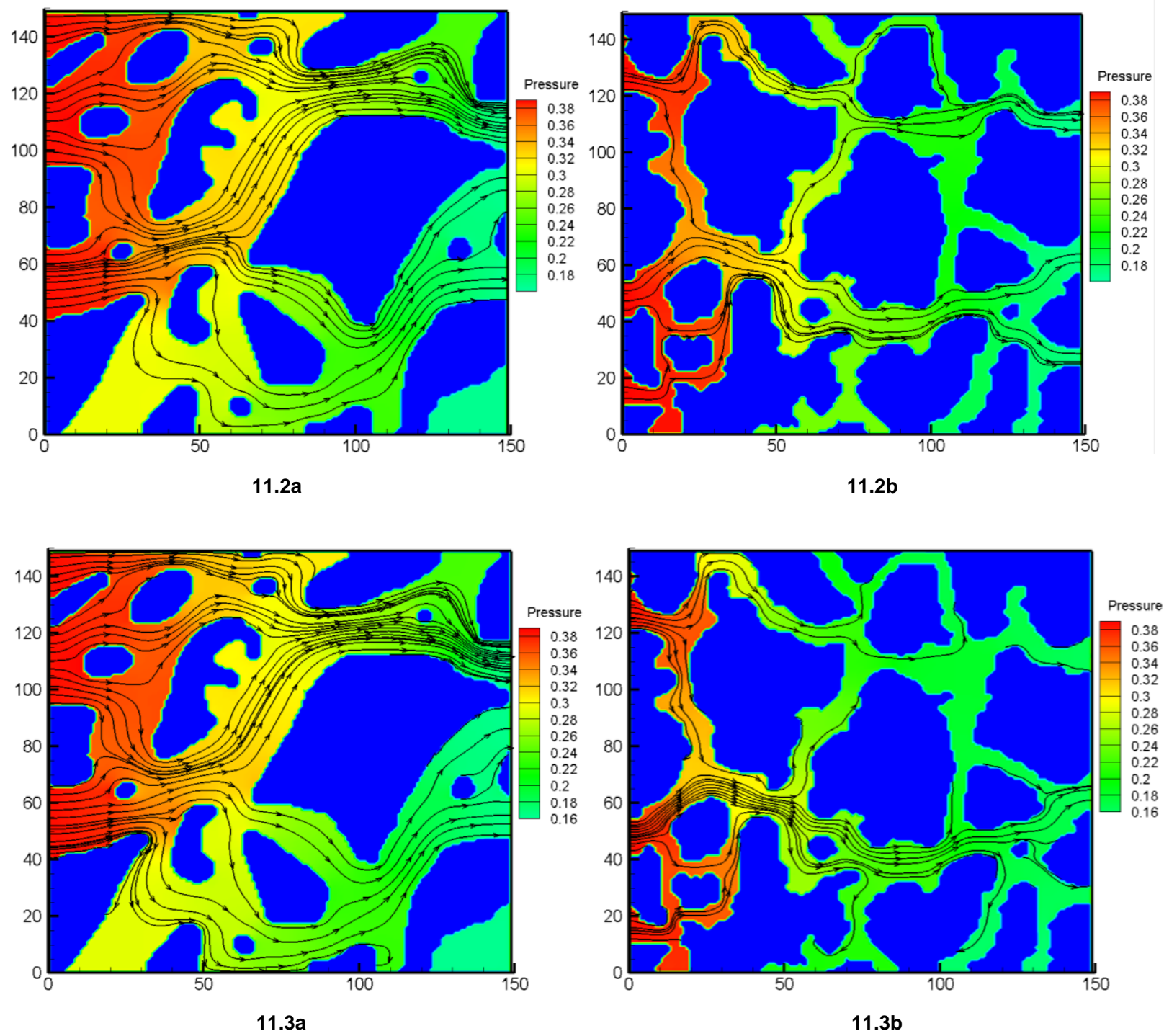

Figure 11: Pore structures and streamlines for $\epsilon=0.6$ and $\epsilon=0.3$ 


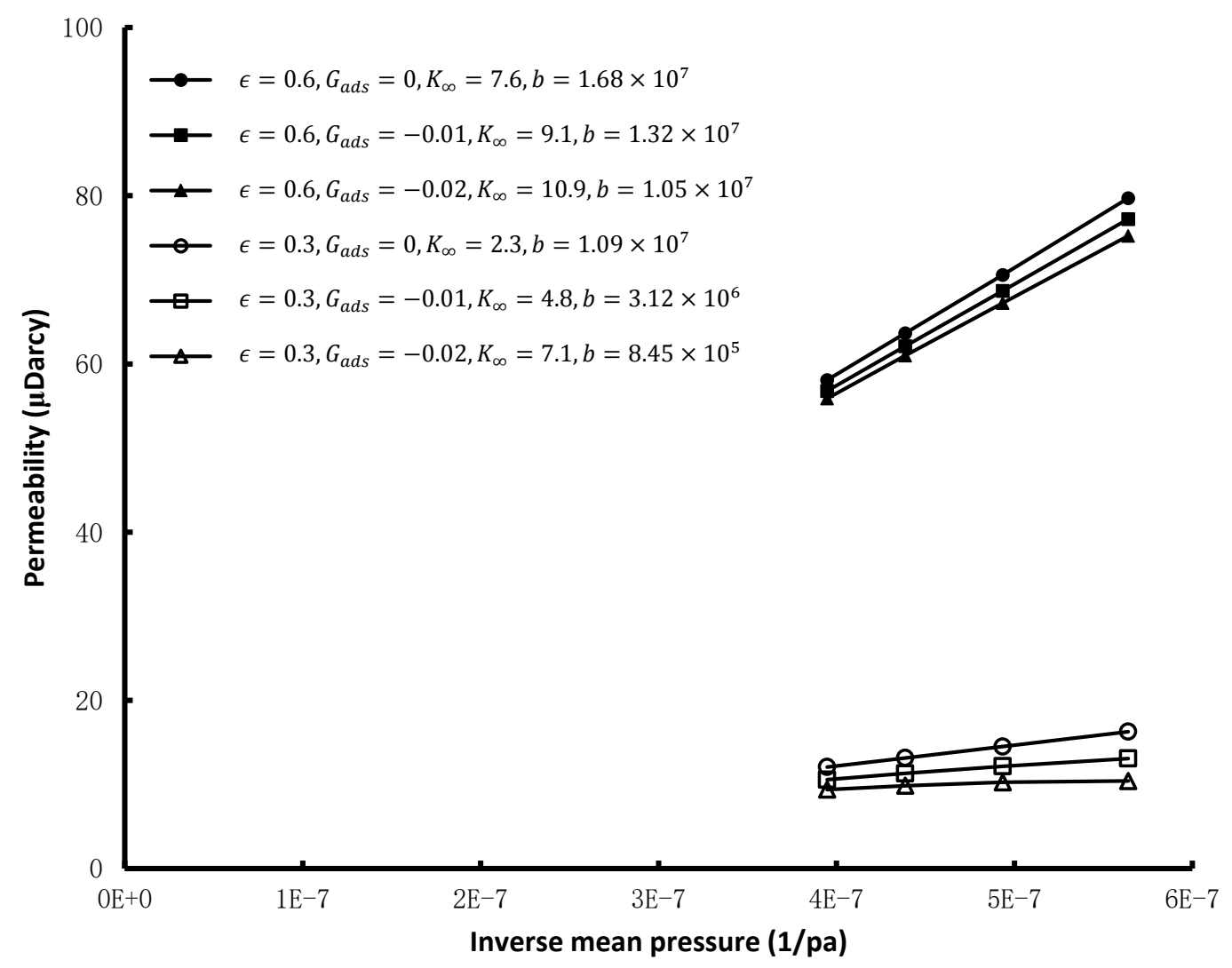

Figure 12: Gas permeability for $\epsilon=0.6$ and $\epsilon=0.3$ with different $G_{a d s}$

\section{Conclusions}

In shale formations, the majority of natural gas is stored in micro-scale and nanoscale organic-rich kerogen pores. The characteristics of the extremely small organic pores prevent many conventional numerical techniques from effectively modeling gas flows in shale formations. Moreover, in kerogen a large amount of natural gas has been claimed to be stored as adsorbed gas coexisting with free gas. Gas flows due to the coexistence of adsorbed and free gas have very different dynamics from conventional gas flows. In addition, gas flow within kerogen pores falls into either the slip flow regime or the transitional flow regime $0.001<K_{n}<10$, in which the non-slip boundary condition no longer holds. In this regard, we have presented the kinetic-based generalized lattice Boltzmann method (LBM). In generalized LBM, the selection of the relaxation times was adjusted to solve flows in the micro systems where the Knudsen layer plays an important role. In addition, combined-bounce back specular-reflection (CBBSR) boundary conditions have been adopted in order to capture the gas slippage effect. We have incorporated the longrange intermolecular forces between the adsorbed gas and the free gas and the adsorptive forces between the organic pore walls and the nearest fluid molecules into the generalized LBM. It is the first time that a 
generalized LBM has been coupled with adsorption to model and simulate natural gas flows in shale formations.

Neglecting the adsorption effects, we have validated our numerical simulation results with existing data for various Knudsen numbers. The gas slippage effect has been clearly observed. As the Knudsen number was increased, the slippage effect became more important near the pore walls. The gas flow phenomena have been investigated in a $2 \mathrm{D}$ organic nanotube with adsorption effects. As the Knudsen number increased, the difference between boundary velocity and bulk velocity became smaller. At a large Knudsen number $\left(K_{n}=0.2 \& 0.5\right)$, surface transport of the adsorbed gas became more important compared with low Knudsen number cases. Methane gas flows in different sizes of organic nanotubes have been simulated and compared with regular micro-flows without adsorption. For a small nanotube $(10 \mathrm{~nm})$ at $T=300 \mathrm{~K} \& P=10 \mathrm{~atm}$, the average flow velocity of methane with a certain amount of adsorption is approximately $37 \%$ smaller than the average flow velocity without adsorption. As the tube size increased, the adsorption effects became less important. The effects for different amounts of adsorption were also investigated. The greater the amount of adsorbed gas contained in the organic nanotube, the smaller the average velocity. Lastly, gas flows driven by a pressure difference in two complex 2D nano-porous structures $(150 \mathrm{~nm} \times 150 \mathrm{~nm})$ were simulated for different adsorptive forces. The gas "permeability" of methane was determined for $\epsilon=0.6$ and $\epsilon=0.3$ porous media and the Klinkenberg constants have been determined for different adsorption effects. The non-linearity effect was observed for the tighter pore structure $(\epsilon=0.3)$ due to the higher-order slippage effect. The "permeability" has the unit of micro-Darcy, which is acceptable because these two porous media have rather high porosity and good pore connectivity compared to real kerogen core data. In the future, a 3D model will be developed and, later, it will be applied to more realistic kerogen pore structures where the true permeability will be predicted.

\section{Acknowledgments}

This research is supported in partial by US National Science Foundation through NSF Grant DMS1209124, Society of Petroleum Engineers - Gulf Coast Section through its distinguish professorship endowment, Institute of Petroleum Exploration \& Development - Langfang Branch, PetroChina Company Limited, and CNPC Chuanqing Drilling Engineering Company Limited. The authors also thank PetroChina Company Limited for the permission to publish this paper. 


\section{References}

Adesida, A.G., Akkutlu, I.Y., Resasco, D.E., Rai, C.S., 30 October-2 November 2011. Characterization of Barnett Shale Kerogen Pore Size Distribution using DFT Analysis and Grand Canonical Monte Carlo Simulations, SPE-147397, SPE Annual Technical Conference and Exhibition, Denver, Colorado, USA.

Ambrose, R.J., Hartman, R.C., Diaz-Campos, M., Akkutlu, I.Y., Sondergeld, C.H., 23-25 Feb 2010. New pore-scale considerations in shale gas in-place calculations, SPE-131772, SPE Unconventional Gas Conference, Pittsburgh, Pennsylvania, USA.

Bhatnagar, P.L., Gross, E.P. and Krook, M., 1954. A model for collision processes in gases. I. Small amplitude processes in charged and neutral one-component systems. Phys. Rev., 94(511).

Chen, H.D., Chen, S.Y. and Matthaeus, W.H., 1992. Recovery of the Navier-Stokes equations using a lattice-gas Boltzmann method. Phys. Rev. A, 45(R5339(R)).

Chen, S. and Tian, Z.W., 2009. Simulation of microchannel flow using the lattice Boltzmann method. Physica A, 388(23), pp. 4803-4810.

d'Humieres, D., 1992. Generalized lattice Boltzmann equations”, In Rarefied gas dynamics: theory and Simulations. AIAA, Volume 159, pp. 450-458.

Fathi, E. and Akkutlu, I.Y., 2013. Lattice Boltzmann method for simulation of shale gas transport in kerogen. SPE Journal, 18(01).

Fathi, E., Tinni, A. and Akkutlu, I.Y., 2012. Correction to Klinkenberg slip theory for gas flow in nanocapilaries. Int. J. Coal Geology, Volume 103, pp. 51-59.

Geier, M., Greiner, A. and Korvink, J.G., 2006. Cascaded digital lattice Boltzmann automata for high Reynolds number flow. Phys. Rev. E, 73(066705).

Ghanizadeh, A., Bhowmik, S., Haeri-Ardakani, O., Sanei, H., Clarkson, C.R., 2015. A Comparison of Shale Permeability Coefficients Derived Using Multiple Non-steady-state Measurement Techniques: Examples from the Duvernay Formation, Alberta (Canada). Fuel, Volume 140, pp. 371-387.

Ginzburg, I. and d'Humieres, D., 2003. Multireflection boundary conditions for lattice Boltzmann models. Phys. Rev. E, 68(066614).

Ginzburg, I., 2005. Equilibrium-type and link-type lattice Boltzmann models for generic advection and anisotropic-dispersion equation. Advances in Water Resources, 28(11), pp. 1171-1195.

Guo, Z.L., Shi, B.C. and Zheng, C.G., 2007. An extended Navier-Stokes formulation for gas flows in the Knudsen layer near a wall. Europhys. Lett., 80(24001).

Guo, Z.L., Zhao, T.S. and Shi, Y., 2006. Physical symmetry, spatial accuracy, and relaxation time of the lattice Boltzmann equation for microgas flows. J. Appl. Phys., 99(074903). 
Guo, Z.L., Zheng, C.G., and Shi, B.C., 2008. Lattice Boltzmann equation with multiple effective relaxation times for gaseous microscale flow. Phys. Rev. E, 77(036707).

He, X.Y. and Doolen, G.D., 2002. Thermodynamic foundation of kinetic theory and lattice Boltzmann models for multiphase flows. J. Stat. Phys., 107(1/2), pp. 309-328.

Heller, R., Vermylen, J. and Zoback, M., 2014. Experimental investigation of matrix permeability of gas shales. AAPG Bullentin, 98(5), pp. 975-995.

Kang, S.M., Fathi, E., Ambrose, R.J., Akkutulu, I.Y., Sigal, R.F., 2011. Carbon Dioxide Storage Capacity of Organic-Rich Shales. SPE Journal, 16(04), pp. 842-855.

Wei, C.J., Qin, G., Guo, W., Yan, B.C., Guo, W., Killough, J.E., Wang, H.Y., Liu, H.L. 19-25 April 2013. Characterization and analysis on petrophysical parameters of a marine shale gas reservoir. SPE Western Regional \& AAPG Pacific Section Meeting 2013 Joint Technical Conference, Monterey, California, USA.

Klinkenberg, L.J., 1941. The permeability of porous media to liquids and gases. API Drilling and Production Practice, 41(200).

Lallemand, P. and Luo, L.S., 2000. Theory of the lattice Boltzmann method: Dispersion, dissipation, isotropy, Galilean invariance, and stability. Phys. Rev. E, 61(6546).

Li, Q., He, Y.L., Tang, G.H. and Tao, W.Q., 2011. Lattice Boltzmann modeling of microchannel flows in the transition flow regime. Microfluidics and Nanofluidics, 10(3), pp. 607-618.

Martys, N.S. and Chen, H.D., 1996. Simulation of multicomponent fluids in complex three-dimensional geometries by the lattice Boltzmann Method. Phys. Rev. E, 53(743).

Meng, J.P., Dongari, N., Reese, J.M., Zhang, Y.H., 2012. Beyond the Knudsen number: assessing thermodynamic non-equilibrium in gas flows. In progress.

Moghadam, A.A. and Chalaturnyk, R., 2014. Expansion of the Klinkenberg's slippage equation to low permeability porous media. Int. J. Coal Geology, Volume 123, pp. 2-9.

Niu, X.D., Hyodo, S.A., Munekata, T., and Suga, K., 2007. Kinetic lattice Boltzmann method for microscale gas flows: Issues on boundary condition, relaxation time, and regularization. Phys. Rev. E, 76(036711).

Ohwada, T., Sone, Y. and Aoki, K., 1989. Numerical analysis of the Poiseuille and thermal transpiration flows between two parallel plates on the basis of the Boltzmann equation for hard-sphere molecules. Phys. Fluids A, 1(12), p. 2042.

Premnath, K.N. and Abraham, J., 2007. Three-dimensional multi-relaxation time (MRT) latticeBoltzmann models for multiphase flow. J. Comp. Phys., 224(2), pp. 539-559.

Premnath, K.N. and Banerjee, S., 2009. Incorporating forcing terms in cascaded lattice-Boltzmann approach by method of central moments. Phys. Rev. E, 80(036702). 
Premnath, K.N., Pattison, M.J. and Banerjee, S., 2009. Generalized lattice Boltzmann equation with forcing term for computation of wall-bounded turbulent flows. Phys. Rev. E, 79(026703).

Qian, Y.H., Succi, S. and Orszag, S.A., 1995. Recent advances in lattice Boltzmann computing. Ann. Rev. Comp. Phys., Volume 30, pp. 195-242.

Ren, J.J., Guo, P., Guo, Z.L. and Wang, Z.H., 2015. A lattice Boltzmann model for simulating gas flow in kerogen pores. Tranp. in Porous Media, 106(2), pp. 285-301.

Sbragaglia, M. and Succi, S., 2005. Analytical calculation of slip flow in lattice Boltzmann models with kinetic boundary conditions. Phys. Fluids, 17(093602).

Scheidrgger, A., 1972. The physics of flow through porous media, 3rd ed.. Toronto, Canada: University of Toronto Press.

Shan, X.W. and Chen, H.D., 1993. Lattice Boltzmann model for simulating flows with multiple phases and components. Phys. Rev. E, 47(3).

Shan, X.W., Yuan, X.F. and Chen, H.D., 2006. Kinetic theory representation of hydrodynamics: a way beyond the Navier-Stokes equation. J. Fluid Mech., Volume 550, pp. 413-441.

Stops, D.W., 1969. The mean free path of gas molecules in the transition regime. J. Phys. D: Appl. Phys., $3(685)$.

Suga, K., Takenaka, S., Ito, T., Kaneda, M., Kinjo, T. and Hyodo, S., 2010. Evaluation of a lattice Boltzmann method in a complex nanoflow. Phys. Rev. E, 82(016701).

Sukop, M.C. and Or, D., 2004. Lattice Boltzmann method for modeling liquid-vapor interface configurations in porous media. Water Resources Res., 40(1).

Sukop, M.C. and Thorne Jr., D.T., 2006. Lattice Boltzmann Modeling: an introduction for geoscientists and engineers., New York Springer.

Tang, G.H., Tao, W.Q. and He, Y.L., 2005a. "Lattice Boltzmann method for gaseous microflows using kinetic theory boundary conditions. Phys. Fluids, 17(058101).

Tang, G.H., Tao, W.Q. and He, Y.L., 2005b. Gas Slippage effect on microscale porous flow using the lattice Boltzmann method. Phys. Rev. E, 72(056301).

Whitaker, S., 1986. Flow in porous media I: A theoretical derivation of Darcy's law. Transport in Porous Media, 1(1), pp. 3-25.

Yu, H.D., Girimaji, S.S. and Luo, L.S., 2005. Lattice Boltzmann simulations of decaying homogeneous isotropic turbulence. Phys. Rev. E, 71(016708).

Zamirian, M., Aminian, K., Ameri, S. and Fathi, E., 30 September- 2 October 2014. New steady-state technique for measuring shale core plug permeability. SPE/CSUR Unconventional Resources ConferenceCanada, Calgary, Alberta, Canada. 
Zhang, X.L., Xiao, L.Z., Shan, X.W. and Guo, L., 2014. Lattice Boltzmann simulation of shale gas transport in organic nano-pores. Scientific Reports, 4(4843).

Zhang, Y.H., Gu, X.J., Barber, R.W., and Emerson, D.R., 2006. Capturing Knudsen layer phenomena using a lattice Boltzmann model. Phys. Rev. E, 74(046704).

Zhu, G.Y., Liu, L., Yang, Z.M., Liu, X.G., Guo, Y.G., Cui, Y.T., Aug 15-19 2007. Experiment and mathematical model of gas flow in low permeability porous media. Fifth International Conference on Fluid Mechanics, Shanghai, China.

Ziarami, A.S. and Aguilera, R., 2012. Knudsen's permeability correction for Tight Porous Media. Tranp. Porous Med., Volume 91, pp. 239-260. 